\title{
A microgenetic study of insightful problem solving is
}

\author{
Koen Luwel ${ }^{\mathrm{a}, *}$, Robert S. Siegler ${ }^{\mathrm{b}}$, Lieven Verschaffel ${ }^{\mathrm{a}}$

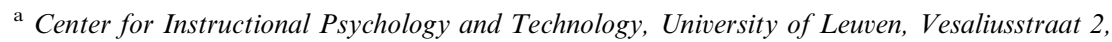 \\ B-3000 Leuven, Belgium \\ b Department of Psychology, Carnegie Mellon University, Pittsburgh, PA 15213, USA
}

Received 30 May 2007; revised 25 July 2007

Available online 4 October 2007

\begin{abstract}
An eight-session microgenetic study of acquisition of an insightful problem-solving strategy was conducted. A total of 35 second graders who did not use this insightful strategy initially were assigned to two groups that differed in the frequency of problems likely to facilitate discovery and generalization of the strategy. Children in the facilitative problems group discovered the insightful strategy earlier, used it more often subsequently, and transferred it more often to novel problems than did those in the nonfacilitative problems group. Children generally discovered the insightful strategy on the most facilitative items and extended it progressively to items on which its advantages were smaller but still substantial. The results indicate that experience outside the experimental situation, as well as experience inside the experimental situation, influences use of new strategies.

(C) 2007 Elsevier Inc. All rights reserved.
\end{abstract}

Keywords: Strategies; Microgenetic method; Mathematics/Numbers; Problem solving; Cognitive development

\footnotetext{
Koen Luwel is a Postdoctoral Fellow of the Fund for Scientific Research-Flanders (Belgium). This study was funded by GOA Grant 2006/1 from the Research Fund K.U. Leuven, Belgium, to the Center for Instructional Psychology and Technology and by U.S. National Institutes of Health Grant HD 19011. The authors thank the second-grade teachers and pupils as well as the principals of the "Heilig Hart" and "Klim-Op" primary schools in Sint-Truiden, Belgium. Thanks are also due to David Bjorklund and two anonymous reviewers for their helpful comments on earlier drafts of this article.

* Corresponding author.

E-mail address: Koen.Luwel@ped.kuleuven.be (K. Luwel).
} 


\section{Introduction}

Understanding how change occurs is among the deepest challenges facing the field of cognitive development. The challenges are both conceptual and methodological. The main conceptual difficulty is that understanding change implies not only describing children's thinking at different points in development but also explaining how and why the changes between the different states occur. Generating well-grounded answers to these "how" and "why" questions is not a trivial task. The main methodological difficulty is that standard cross-sectional and longitudinal designs are of limited usefulness for understanding change. The density of observations that these traditional developmental methods yield is too low to answer many basic questions about change (Adolph, Robinson, Young, \& Gill-Alvarez, 2007; Demetriou \& Raftopoulos, 2004; Kuhn, 1995; Miller \& Coyle, 1999).

The microgenetic method provides a means for obtaining the kind of fine-grained information that is essential for understanding the change process. This method emphasizes high-density sampling of changing competence while the change is occurring. A common form of the method involves accelerating the change process by providing participants with experiences intended to promote discovery of more advanced concepts, strategies, rules, or theories. The increased density of opportunities to discover and exercise the new understanding allows more detailed examination of change than would otherwise be possible.

Microgenetic designs incorporate three features. First, observations encompass the period of rapid change, starting before rapid change begins and continuing until the competence has reached a relatively advanced state. Second, density of observations is high relative to the rate of change of the phenomenon under study. Third, observations are examined on a trial-by-trial basis with the goal of understanding the process that gives rise to both qualitative and quantitative aspects of change.

The microgenetic method has proved to be applicable to many content domains and age groups. These include infants' and toddlers' locomotion and tool use (Adolph, 1997; Chen \& Siegler, 2000); preschoolers' simple addition, controlled attention, and theory of mind (Amsterlaw \& Wellman, 2006; Miller \& Aloise-Young, 1995; Siegler \& Jenkins, 1989); school-age children's memory and language (Coyle \& Bjorklund, 1997; Karmiloff-Smith, 1992; Schlagmüller \& Schneider, 2002); and adolescents' scientific reasoning and problem solving (Kuhn, Garcia-Milla, Zohar, \& Andersen, 1995; Schauble, 1996).

Microgenetic methods also allow examination of five dimensions of change: its path, rate, breadth, source, and variability. The path of change refers to the sequence of knowledge states that lead to advanced understanding or skill. The rate of change includes both the amount of time or experience required for the new strategy to be used initially and the amount of time or experience required for it to be used consistently on the type of problems on which it was discovered; the first type is referred to as the strategy's rate of discovery, and the second type is referred to as its rate of uptake. The breadth of change is the range of problems to which the new approach is generalized. The source of change refers to the experiences that set the change in motion. The variability of change concerns individual differences in the other dimensions of change.

In the current study, we used a microgenetic design to examine the discovery and generalization of an insightful quantification strategy. The task was to determine the 
number of blocks (green squares) in $10 \times 10$ grids. Previous studies of this task by Luwel, Verschaffel, Onghena, and De Corte (2000), Luwel, Verschaffel, Onghena, and De Corte (2001) and by Verschaffel, De Corte, Lamote, and Dherdt (1998) revealed that children solve such problems via two main strategies: addition and subtraction. The addition strategy involves starting at 1 and enumerating blocks until all are included in the count or estimate. This approach is most efficient when there are relatively few blocks to enumerate. The subtraction strategy involves either starting at 100 and counting down the number of empty squares or counting the number of empty squares and subtracting that number from 100. It is most efficient when there are nearly 100 blocks. Thus, the efficiency of the two strategies varies strongly, but inversely, with the number of blocks in the grid.

For any given amount of counting, the subtraction strategy in both of its forms imposes greater cognitive demands than does the addition strategy. Counting backward from 100 is less frequent and less well learned than counting forward from 1. Counting the number of empty squares and subtracting that number from 100 requires two operations rather than one (counting and subtracting rather than counting only). Both the relative unfamiliarity with counting backward and the extra operation probably contribute to the subtraction strategy for solving grid problems emerging later in development than the addition strategy (Luwel, Verschaffel, Onghena, \& De Corte, 2000; Luwel, Verschaffel, Onghena, \& De Corte, 2001; Verschaffel et al., 1998). These previous studies have also shown that use of the subtraction strategy becomes faster and more accurate with age and that the adaptiveness of choices between the subtraction strategy and the addition strategy also improves (see also Luwel, Lemaire, \& Verschaffel, 2005). However, the cross-sectional designs used in the previous studies did not allow detailed analysis of how the subtraction strategy emerges and how it is generalized to novel problems.

The current study applied a microgenetic design to obtain a fine-grain depiction of acquisition of the subtraction strategy. ${ }^{1}$ Together with trial-by-trial analyses of strategy use, this design allowed identification of the trial of discovery of the subtraction strategy, which in turn allowed analysis of the path, rate, breadth, and variability of the acquisition. The design also made it possible to examine a potential source of change, namely, encountering numerous problems where the subtraction strategy would be much more efficient than the addition strategy.

The grid task had the advantage of providing a straightforward and continuous metric for analyzing the influence of the type of problem on discovery and generalization of a new strategy. For example, the task allowed examination of whether children discovered the subtraction strategy on items on which its advantages, relative to the addition strategy, were particularly large (items with nearly 100 squares) and whether its generalization beyond its initial use occurred quickly and broadly, slowly but eventually broadly, only narrowly, or in an unsystematic pattern.

\footnotetext{
${ }^{1}$ In using the expression "acquisition/discovery of the subtraction strategy" we do not want to suggest that the children in our study had no prior knowledge (conceptual, procedural, or factual) of subtraction or that they never applied this knowledge in other contexts (e.g., solving a symbolically presented missing addend sum as $2+$ $\ldots=9$ or a corresponding word problem as "Pete had 2 apples. Ann gave him some more. Now Pete has 9 apples. How many apples did he get from Ann?" by directly subtracting 2 from 9). Rather, this expression refers to the first time children used the subtraction strategy in the current task.
} 
To achieve these goals, we presented second graders who did not use the subtraction strategy initially with eight sessions of experience with grid problems. In the four practice sessions (Sessions 2, 3, 5, and 6), children encountered either a high or low percentage of facilitative items, that is, items on which the advantages of the subtraction strategy were especially large. Children in the facilitative problems group were presented with $90 \%$ of items with 80 to 99 squares in the four practice sessions; encountering many such items was expected to promote discovery and generalization because on these items the subtraction strategy should be much faster and more accurate than the addition strategy. Children in the nonfacilitative problems group were presented with only $10 \%$ of such items in the practice sessions.

In the three test sessions (Sessions 1, 4, and 7), all children were presented with identical problems that were distributed evenly across the range 1 to 99 ; thus, $10 \%$ of test session problems involved 90 to 99 blocks, $10 \%$ involved 80 to 89 blocks, and so forth. The test sessions allowed examination of whether encountering a high percentage of facilitative problems in the practice sessions increased the use of the subtraction strategy even when the context of other problems in the session was not facilitative. Finally, in the transfer session (Session 8), children were presented with a different grid from the one used in the other seven sessions to assess generalization of the strategy to related but different problems.

The main hypotheses can be organized around the five dimensions of change described earlier. With regard to the path of change, we expected that in both experimental groups discovery of the subtraction strategy would occur primarily on highly facilitative problems (those with nearly 100 squares). From there, children would progressively extend the new strategy to problems with fewer squares. This prediction follows from previous findings that use of new strategies is quite adaptive even when novel strategies are just beginning to be used (e.g., Lemaire \& Siegler, 1995). With regard to the source of change, we hypothesized that encountering a higher percentage and number of facilitative problems in the practice sessions would increase use of subtraction due to greater activation of the strategy. (For data consistent with this assumption, see Siegler \& Stern, 1998; for a model that illustrates how activation influences strategy use, see Siegler \& Araya, 2005.) With respect to the rate of change, we expected that children who received a high frequency of facilitative items would discover the subtraction strategy more rapidly than would children who received a low frequency of facilitative items (again through greater activation of the strategy). With respect to the breadth of change, we expected that use of the subtraction strategy in the facilitative problems group would extend to a broader range of problems than in the nonfacilitative problems group (again because of greater activation of the strategy). With regard to the variability of change, we expected children to differ considerably in both the rate of discovery and the breadth of generalization of the new strategy but to follow similar paths of learning. This pattern has been typical in previous microgenetic studies (Siegler, 2006).

\section{Method}

\section{Participants}

The initial sample consisted of 74 second graders (40 girls and 34 boys) with a mean age of 7.44 years $(S D=0.47$, range $=6.54-9.38)$. The children came from two schools 
in the Flemish part of Belgium, and the schools were similar in terms of general educational approach, mathematics curriculum, and students' socioeconomic status. All children were tested during October and November, a time when they were in the midst of exploring the number range up to 100 and had already learned to subtract from numbers larger than 20.

Test Session 1 provided pretest data on children's knowledge of the subtraction strategy. Overt behavior, verbal protocols, solution times, and error rates indicated that slightly fewer than half of the children (35 of 74) used the subtraction strategy at least once and thus were excluded from participation in the study. Another 3 children were excluded because they were unable to count to 100; this prevented them from executing the addition strategy accurately and almost certainly also would have prevented them from executing the subtraction strategy accurately. In addition, 1 child was excluded because of behavioral problems (she wandered around and did not stop talking about irrelevant topics). This left a final sample of 35 children ( 22 girls and 13 boys) who participated in all eight sessions. These children were randomly assigned to either the nonfacilitative problems group (18 children) or the facilitative problems group (17 children). The mean ages of the children in the nonfacilitative and facilitative problems groups were 7.55 years $(S D=0.59)$ and 7.46 years $(S D=0.54)$, respectively. The mean general math achievement scores on the Leerlingvolgsysteem (LVS) test (Dudal, 2001), which is normed on the Flemish school population, were $44.33(S D=6.24)$ and $45.43(S D=8.11)$, with a maximum of 60 , for nonfacilitative and facilitative problems groups, respectively. In addition, $t$ tests did not reveal a significant difference between the two groups on any of these measures, all $t \mathrm{~s}<.50$.

\section{Materials}

The task was presented on a PC equipped with a Pentium III processor and a 15 -inch monitor with a screen resolution of $800 \times 600$ pixels. Stimuli were presented within a $10 \times 10$-cm matrix of $1 \times 1-\mathrm{cm}$ squares. These squares were either "on" (filled with a green block) or "off" (having the same black color as the background). The green blocks were located randomly within the $10 \times 10$ grid. Thin red lines separated the rows and columns of the grid, and the border of the grid was also red.

\section{Design and procedure}

At the beginning of Test Session 1, children were seated approximately $50 \mathrm{~cm}$ from the computer monitor. They were told that they would be presented with a number of screen displays, each showing a grid with a different number of green blocks, and that their task was to determine the number of green blocks as accurately as possible. Children also were told that each grid contained 10 rows, that each row contained 10 squares, and that therefore the maximum number of green blocks in each grid was 100. The experimenter also said that children could determine the number of blocks in whatever way they wanted but should not guess, an instruction that was repeated at the beginning of each session. No mention was made of speed; given the large difference in the speed of the addition and subtraction strategies for many numerosities, speed seemed likely to be its own reward. 
To accustom children to the task, three practice trials with 9, 26, and 37 blocks were presented at the beginning of Test Session 1. On these and all subsequent trials, children were asked to state the answer as soon as they knew it. Then the experimenter pressed a key that stopped the computer timer, entered the children's response, and asked the children how they had solved the problem. After this, a new trial began with a different grid appearing on the screen. Each session was video-recorded to allow accurate classification of the strategies.

Children were presented with $2010 \times 10$ grids in each of the first seven sessions, and in the transfer session (Session 8) they were presented with $65 \times 10$ grids. Each grid within a session had a different number of green squares, and the 20 grids within a session were presented in the same random order for each participant to ensure that all children underwent exactly the same learning experience. In both experimental groups, Sessions 1, 4, and 7 were test sessions; Sessions 2, 3, 5, and 6 were practice sessions; and Session 8 was the transfer session.

\section{Test sessions}

In the three test sessions, children were presented with the same 20 grids, with two numerosities from each of the 10 decades from 1 to 100 . Because these test sessions were aimed mainly at comparing the generalization of the subtraction strategy at several points during the microgenetic study, the same 20 items were used in each test session to maximize comparability of results from the different test sessions. Moreover, because having solved a specific item with a particular strategy might affect the strategy choice on the next item (Siegler \& Araya, 2005), these 20 items were presented in the same order in all test sessions. Although it was theoretically possible for the second graders to memorize the number of squares in particular arrays, their consistently long solution times and/or verbal reports indicated that they did not do so.

\section{Practice sessions}

In the four practice sessions, the items that were presented differed in the two experimental groups. In the facilitative problems group, $90 \%$ of the numerosities in each session were randomly selected from the range 80 to 99 , where the subtraction strategy was expected to generate answers more efficiently. The remaining $10 \%$ of numerosities were randomly chosen from the range 1 to 50 , so that the children would have some experience with problems on which the addition strategy was likely to be faster and more accurate.

In the nonfacilitative problems group, $90 \%$ of the numerosities in each practice session were randomly chosen from the range 1 to 50 , where the addition strategy was expected to be faster and more accurate. The remaining $10 \%$ of numerosities were randomly selected from the range 80 to 99 , so that children in this group would also have some experience with problems on which the subtraction strategy was more efficient.

In both groups, the particular numerosities with which children were presented varied across the four practice sessions. However, within a given practice session of a given experimental group, all children were presented with the same numerosities in the same orderan order that was random except for the restriction that the two atypical numerosity items in each session always were presented on the 5 th and 15 th trials of the session. 


\section{Transfer session}

Like the test sessions, the transfer session involved presentation of the same items in the same order to children in both experimental groups. However, the transfer problems involved a $5 \times 10$ grid rather than the $10 \times 10$ grid used in Sessions 1 to 7 . The numerosities on all six trials in the transfer session were randomly selected from the range 35 to 50 , a range that was expected to facilitate use of the subtraction strategy to varying degrees (e.g., more for 48 squares than for 36 squares). As in the test sessions, children were told the maximum number of squares that this grid could contain (50). Appendix A provides a list of the number of squares presented on each problem in each session.

Children were tested individually for eight sessions, with two sessions per week (Week 1: Test Session 1 and Practice Session 1; Week 2: Practice Session 2 and Test Session 2; Week 3: Practice Session 3 and Practice Session 4; and Week 4: Test Session 3 and Transfer Session); thus, the duration of the study was 4 weeks for each participant except in cases of illness or schedule conflicts.

\section{Results}

The results are presented in four main sections. In the first section, we provide an overview of changes in performance over the course of the study. Next, we examine changes in performance over the three test sessions, a comparison that allows rigorous tests of the effects of encountering facilitative or nonfacilitative problems. After that, we examine discovery and generalization of the subtraction strategy in the practice sessions of children in the facilitative problems group; this group received enough problems on which use of the subtraction strategy was highly advantageous to allow detailed microgenetic analyses of the discovery and generalization processes. Finally, we analyze individual differences in discovery and generalization of the subtraction strategy among children in the facilitative problems group, again because this group had received enough relevant problems to gain in-depth understanding. Tukey's HSD tests were used in all post hoc comparisons.

\section{Overview}

Based on the children's overt behavior and verbal reports on each trial, we identified 12 procedures that children used to solve the grid problems. Of these 12 procedures, 2 were common variants of the addition strategy: counting the green blocks upward from 1 by ones (60\% of trials) and counting upward from 1 by twos $(9 \%)$. In addition, 2 procedures were common variants of the subtraction strategy: the counting down procedure, where the number of empty squares was counted down from 100 (12\% of trials), and the direct subtraction procedure, which involved counting the number of empty squares and then subtracting that number from $100(9 \%)$. The other 8 procedures were together used on $10 \%$ of trials; all except guessing were variants of the addition strategy (e.g., counting upward by a combination of ones and twos and counting complete rows by tens and adding 1 for each square in incomplete rows or columns). Over the eight sessions, the number of procedures used by each child ranged from one to eight with a mean of 3.22 $(S D=0.88)$. Fully $85 \%$ of the children used two to four of the procedures. In all subsequent analyses, we grouped together as the subtraction strategy the two variants of that 


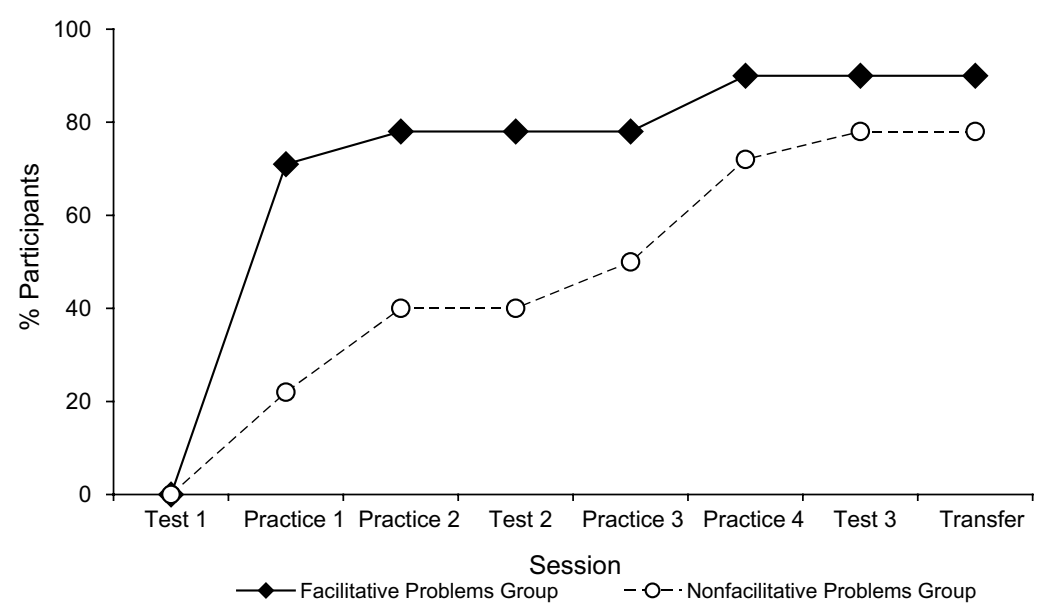

Fig. 1. Cumulative percentages of children who had discovered the subtraction strategy by each session in the two experimental groups.

approach and grouped together as the addition strategy all other approaches except guessing (because all involved proceeding upward from 1 ). ${ }^{2}$

Perhaps the most striking finding from the experiment was how strongly item characteristics influenced discovery of the subtraction strategy. All 29 children who ever used the subtraction strategy first used it on a problem with 93 to 99 blocks. Such items constituted only $7 \%$ of trials in the nonfacilitative problems group and $21 \%$ of trials in the facilitative problems group. This means that every child first used the subtraction strategy on an item where it required little counting down from 100 and thus was far more efficient than the addition strategy. In both groups, the average number of green blocks on the trial where the subtraction strategy was discovered was 96.

Encountering facilitative or nonfacilitative problems in the practice sessions also influenced discovery of the subtraction strategy. Fig. 1 presents the cumulative percentages of children who had used the subtraction strategy at least once by a given session. In the facilitative problems group, more than $70 \%$ of children discovered the subtraction strategy in Practice Session 1, which was the first session in which discovery was possible (children who used the strategy in Test Session 1 did not participate further in the experiment).

\footnotetext{
${ }^{2}$ Our initial plan was to conduct an in-depth analysis of the development of the two subtraction variants during the course of the microgenetic study. With this analysis, we wanted to test the expectation that children would gradually exchange the simpler variant of the subtraction strategy (i.e., counting down) for the more advanced one (i.e., direct subtraction). We found, however, that a large majority of the children (i.e., 23 of 29) exclusively used one of the two variants of the subtraction strategy throughout the study, with 13 children solely using the "counting down" variant and 10 children exclusively applying the "direct subtraction" variant. Only 6 children used both subtraction strategies. The analysis of the development of the use of both variants of the subtraction strategy in this latter group of children did not confirm the above-mentioned expectation, most likely as a result of the limited number of participants in this group. All research questions that are included in the remainder of the Results section were examined separately for the two groups of children who exclusively applied either the counting down or direct subtraction variant of the subtraction strategy. None of these analyses yielded a significant effect with respect to the subtraction variants. Because of the absence of any effect of the two variants of the subtraction strategy, we decided to lump them together and treat them as a single strategy.
} 
As illustrated in Fig. 1, fewer than $30 \%$ of children in the nonfacilitative problems group discovered the strategy in that session. More generally, the cumulative percentage of children who had discovered the subtraction strategy increased more gradually in the nonfacilitative problems group than in the facilitative problems group, although by Practice Session 4 the percentage of discoverers in the two groups did not differ. A Kolmogorov-Smirnov two-sample test showed that children in the facilitative problems group discovered the subtraction strategy after fewer sessions, $D_{14,15}=102.90, p<05$.

To determine whether the higher density of facilitative problems in the facilitative problems group led to discovery of the subtraction strategy after fewer such problems, we then compared the numbers of items with between 93 and 99 blocks that were presented before children discovered the subtraction strategy. (In this analysis, children who never discovered the subtraction strategy were assigned the total number of items with 93-99 blocks plus one). The analysis did not show any difference between the two groups in the number of facilitative problems prior to discovery of the subtraction strategy; the number of such problems averaged $4.06(S D=3.42)$ in the nonfacilitative problems group and 4.52 $(S D=3.83)$ in the facilitative problems group. Thus, the discovery of the subtraction strategy after fewer sessions in the facilitative problems group was due to the larger number of items with few unfilled spaces that were presented to that group rather than to the higher percentage of such problems within the practice sessions.

\section{Changes over test sessions}

\section{Strategy use}

To examine changes in use of the subtraction strategy of children in the two experimental groups on identical problems, we analyzed test session performance of the 29 children who discovered the strategy in the course of the experiment (14 in the nonfacilitative problems group and 15 in the facilitative problems group. A 2 (Group: facilitative or nonfacil-

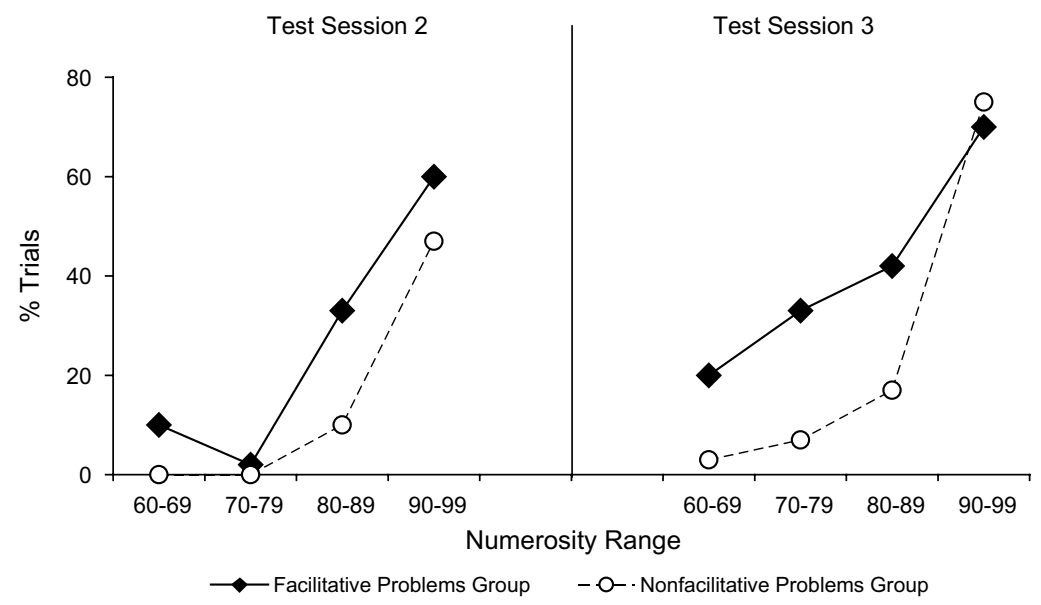

Fig. 2. Percentages of trials solved with the subtraction strategy in four numerosity ranges in Test Sessions 2 and 3. 
itative problems) $\times 2$ (Test Session: 2 or 3$) \times 4$ (Numerosity: $60-69,70-79,80-89$, or 90 $99)^{3}$ ANOVA on number of uses of the subtraction strategy revealed main effects of numerosity, $F(3,81)=38.03, p<0001, \eta^{2}=57$, and session, $F(1,27)=5.91, p<05, \eta^{2}=18$, and a marginally significant effect of group, $F(1,27)=3.36, p=08, \eta^{2}=11$. As shown in Fig. 2, the subtraction strategy was used more on items with 90 to 99 blocks (63\% of trials) than on items with 80 to 89,70 to 79 , and 60 to 69 blocks $(27,16$, and $9 \%$, respectively), more in Test Session 3 than in Test Session 2 (34 vs. 23\%), and more in the facilitative problems group than in the nonfacilitative problems group (36 vs. $20 \%$ ).

Correlations between the number of squares on each problem and the frequency of use of the subtraction strategy on that problem in the test sessions indicated that strategy choices were highly adaptive. The greater the number of squares - that is, the easier the execution of the subtraction strategy and the harder the execution of the addition strategy - the more often children used the subtraction strategy on the problem: $r=.60$, $n=20, p<005$ in the nonfacilitative problems group for Test Session $2 ; r=.65, n=20$, $p<005$ in the nonfacilitative problems group for Test Session $3 ; r=.78, n=20, p<001$ in the facilitative problems group for Test Session 2; and $r=.85, n=20, p<001$ in the facilitative problems group for Test Session 3. The correlations tended to increase over sessions as children gained experience with the subtraction strategy, but the differences were not significant.

\section{Solution times}

Use of the subtraction strategy on trials with many blocks was expected to reduce the time required to solve the items. To test this prediction, we conducted a 2 (Group: facilitative or nonfacilitative problems) $\times 3$ (Test Session: 1, 2, or 3) $\times 4$ (Numerosity: 60-69, $70-79,80-89$, or 90-99 blocks) ANOVA on solution times. The analysis indicated main effects of test session, $F(2,48)=34.99, p<0001, \eta^{2}=56$, and numerosity, $F(3$, $72)=6.94, p<001, \eta^{2}=16$. Solution times decreased between Test Sessions 1 and 2 ( 79.59 vs. $62.37 \mathrm{~s}$ ) and between Test Sessions 2 and 3 (62.37 vs. $46.50 \mathrm{~s})$. Solution times for numerosities 80 to 89 were longer than those for numerosities 60 to 69 and 90 to 99 (72.00 s vs. 56.76 and $56.77 \mathrm{~s}$, respectively).

A Test Session $\times$ Numerosity interaction also was present, $F(6,144)=12.55, p<0001$, $\eta^{2}=34$. As shown in Fig. 3, times decreased considerably between Test Sessions 1 and 2 and between Test Sessions 2 and 3 for the ranges 80 to 89 and 90 to 99 . However, only small nonsignificant reductions were present for the ranges 60 to 69 and 70 to 79 . The interaction could also be viewed from another perspective. In Test Session 1, where by definition children never used the subtraction strategy, solution times were longer when 90 to 99 blocks were present than when 60 to 79 blocks were present. In contrast, in Test Sessions 2 and 3, solution times were shorter when 90 to 99 blocks were present than when 60 to 79 blocks were present because in Test Sessions 2 and 3 children usually used the subtraction strategy when 90 to 99 blocks were present.

The low percentage of use of the subtraction strategy on problems with 60 to 89 blocks seemed to be inefficient in that using the addition strategy required more counting and,

\footnotetext{
${ }^{3}$ We included these ranges in the analysis because the subtraction strategy never was used in any test session on a numerosity smaller than 65 .
} 


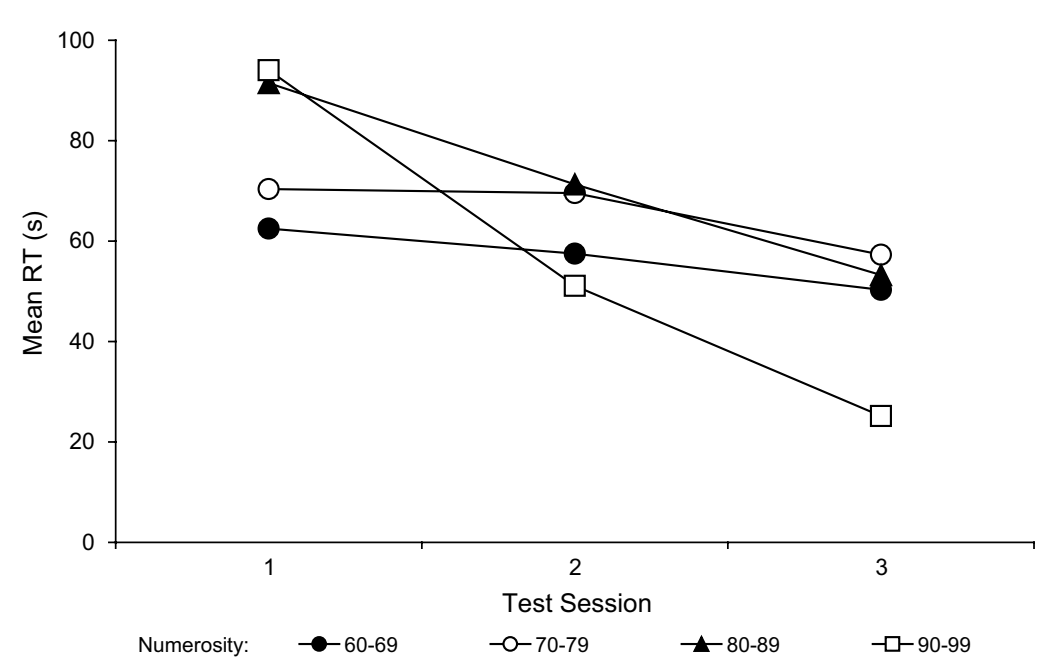

Fig. 3. Mean solution times in each test session for four numerosity ranges.

presumably, more time. However, it was possible that counting down was so much more time-consuming than counting up for any given number of counts that it was actually more efficient to use addition than to use subtraction to solve such problems. Two tests were conducted to test whether this was the case.

One test correlated each child's number of uses of the subtraction strategy on problems where the strategy seemed to be advantageous (those with 60-99 blocks) with the child's mean solution time on those problems. Consistent with the view that greater use of the subtraction strategy led to faster solution times when many blocks were present, the number of test session trials with 60 to 99 blocks that a child solved by using the subtraction strategy correlated substantially with that child's mean solution time on items in the range, $r=-.78, n=29, p<001$ for Test Session 2 and $r=-.72, n=29$, $p<001$ for Test Session 3 .

The second test compared the mean solution time on each item produced by use of the subtraction strategy with the mean solution time produced by use of the addition strategy on the same item. This test indicated that the time saved by using the subtraction strategy was very large. A 2 (Strategy: addition or subtraction) $\times 4$ (Numerosity: 60-69, 70-79, 8089, or 90-99 blocks) ANOVA for Test Session 2 revealed a main effect of strategy, $F(1,15)=$ 108.39, $p<0001, \eta^{2}=88$, and a Strategy $\times$ Numerosity interaction, $F(3,45)=$ 6.94, $p<001, \eta^{2}=32$. Executing the addition strategy took far longer than executing the subtraction strategy on problems with 60 to 99 blocks ( 75.47 vs. 19.65 s). The interaction was due to the difference between mean solution times produced by the two strategies increasing as set size increased: The difference in solution times was $46.13 \mathrm{~s}$ for 70 to 79 blocks, $62.35 \mathrm{~s}$ for 80 to 89 blocks, and $85.15 \mathrm{~s}$ for 90 to 99 blocks (there were too few uses of the subtraction strategy with 60-69 blocks for solution time differences to be meaningful). A parallel analysis for Test Session 3 revealed similar effects of strategy, $F(1$, $10)=29.08, p<0005, \eta^{2}=74$, and of the Strategy $\times$ Numerosity interaction, $F(3$, $30)=8.78, p<0005, \eta^{2}=47$. Again, the interaction between strategy and numerosity was caused by an increase in solution times for the addition strategy and by a decrease 
in solution times for the subtraction strategy between the numerosities 70 to 79 and 80 to 99. The difference in solution times was $32.72 \mathrm{~s}$ for 70 to 79 blocks, $56.08 \mathrm{~s}$ for 80 to 89 blocks, and $56.21 \mathrm{~s}$ for 90 to 99 blocks.

\section{Accuracy}

A 2 (Group: facilitative or nonfacilitative problems) $\times 3$ (Test Session: 1, 2, or 3 ) $\times 4$ (Numerosity: 60-69, 70-79, 80-89, or 90-99 blocks) on percentage of correct answers showed main effects of session, $F(2,48)=15.17, p<0001, \eta^{2}=39$, and numerosity, $F(3,72)=9.74, p<0001, \eta^{2}=29$. Children correctly identified the number of green blocks more often in Test Sessions $2(29 \%)$ and $3(38 \%)$ than in Test Session $1(11 \%)$, and more often on items with 90 to 99 blocks than on items with 80 to 89,70 to 79 , and 60 to 69 blocks ( $40 \%$ vs. 22,16 , and 26\%, respectively).

Test session and numerosity also interacted, $F(6,144)=3.82, p=001, \eta^{2}=14$, due to the number of correct answers increasing over sessions for larger numerosities but not for smaller ones (Fig. 4). For the range 90 to 99, accuracy was greater for Test Session 2 than for Test Session 1 (46 vs. 10\%) and was also greater for Test Session 3 than for Test Session 2 (63 vs. 46\%). Similarly, for the range 80 to 89, accuracy was greater in Test Session 3 than in Test Sessions 2 and 1 (40\% vs. 19 and 6\%, respectively). In contrast, percentage correct did not change over sessions in the ranges 60 to 69 and 70 to 79 .

As with the solution times, we compared the percentage of correct responses on grids with 60 to 99 blocks produced by the addition and subtraction strategies. Paired $t$ tests showed that in Test Session 2, children generated a higher percentage of correct answers on items with 60 to 99 blocks when using subtraction than when using addition (60 vs. $27 \%), t(15)=2.81, p=01, \eta^{2}=35$. A similar result was found for Test Session 3; again, the subtraction strategy generated a higher percentage of correct answers than did the addition strategy (64 vs. $30 \%), t(20)=3.17, p<005, \eta^{2}=33$.

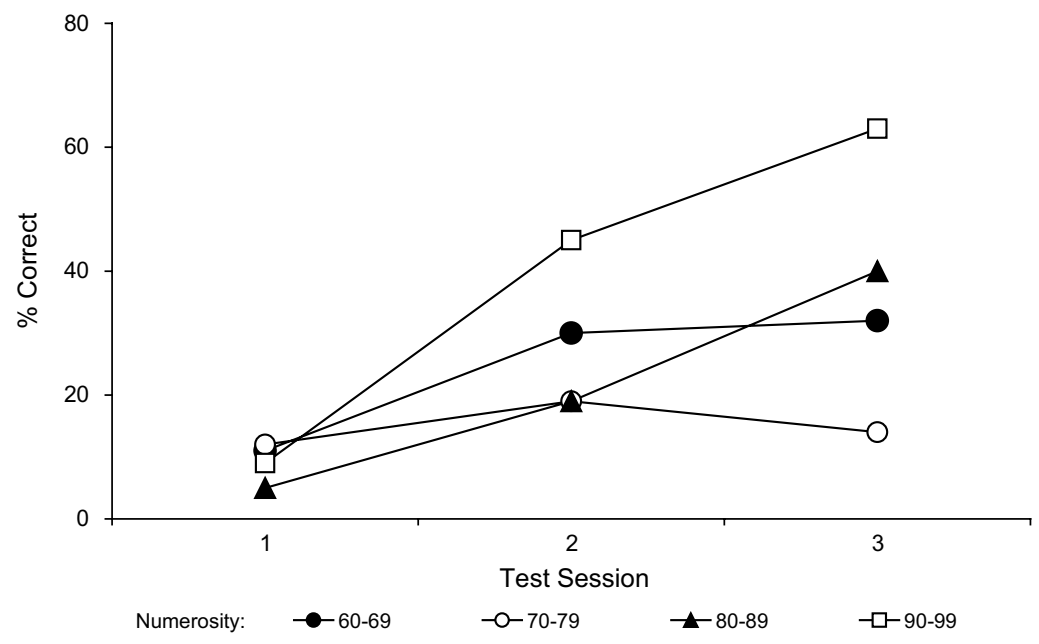

Fig. 4. Percentages correct in test sessions for four numerosity ranges. 


\section{Transfer task performance}

All six transfer problems had blocks in 35 to 49 of the 50 spaces in the grid, and thus could be solved relatively efficiently using the subtraction strategy. The issue was whether the children would extend what they had learned on the $10 \times 10$ grids to the novel problems. A 2 (Group: facilitative or nonfacilitative problems) $\times 3$ (Numerosity: 35-39, 40-44, or 45-49 blocks) ANOVA on use of the subtraction strategy on transfer problems indicated effects of group, $F(1,27)=4.08, \quad p<05, \eta^{2}=13$, and numerosity, $F(2$, $54)=46.07, p<0001, \eta^{2}=63$. Children in the facilitative problems group used the subtraction strategy on a higher percentage of transfer problems than did children in the nonfacilitative problems group (54 vs. $33 \%$ of trials). The subtraction strategy was used on a higher percentage of trials when 45 to 49 blocks were presented than when 40 to 44 blocks were presented (78 vs. $40 \%$ of trials) and on a higher percentage of trials when 40 to 44 blocks were presented than when 35 to 39 blocks were presented (40 vs. $16 \%$ of trials). Thus, participation in the facilitative problems group increased transfer of the subtraction strategy to a different grid, and the absolute amount of use of the subtraction strategy was as great on the transfer problems as on the original ones.

\section{Changes over practice sessions}

This section presents microgenetic analyses of changes in use of the subtraction strategy over the practice sessions. The analysis is limited to the facilitative problems group because only this group had received enough grids with very many blocks to allow detailed analysis of the change process.

\section{Discovery of the subtraction strategy}

We first examined the number of facilitative problems (grids with 80-99 blocks) that children encountered before using the subtraction strategy in each numerosity range. A one-way ANOVA with numerosity range $(80-84,85-89,90-94$, or 95-99) as the sole variable indicated that the more advantageous the problem for the subtraction strategy, the earlier children first used that strategy, $F(3,42)=16.63, p<0001, \eta^{2}=67$. Children used the subtraction strategy earlier on trials with 95 to 99 blocks ( 3.20 such trials) than on trials with 90 to 94,85 to 89 , and 80 to 84 blocks $(8.13,12.13$, and 11.13 such trials, respectively), which did not differ among themselves.

\section{Frequency of use of the subtraction strategy}

To test whether frequency of use of the subtraction strategy progressed gradually from the most advantageous cases to decreasingly advantageous ones, we conducted a 4 (Practice Session: 1,2 , 3, or 4) $\times 4$ (Numerosity: 80-84, 85-89, 90-94, or 95-99) ANOVA on the frequency of use of the subtraction strategy. The analysis revealed main effects of session, $F(3,42)=7.22, p<001, \eta^{2}=34$, and numerosity, $F(3,42)=17.92, p<0001, \eta^{2}=56$. As expected, children used the subtraction strategy on a higher percentage of practice session trials when 95 to 99 blocks were present than when 90 to 94,85 to 89 , and 80 to 84 blocks were present ( $62 \%$ vs. 45,39 , and $39 \%$, respectively). Children also used the subtraction strategy more often in Practice Session 4 than in Practice Sessions 2 and 1 (62\% vs. 40 
and 29\%, respectively) and more often in Practice Session 3 than in Practice Session 1 (54 vs. $29 \%$ ).

A one-way ANOVA was conducted to test more precisely the hypothesis that use of the subtraction strategy was extended gradually from the most advantageous cases to decreasingly advantageous ones. This analysis examined the range of numerosities over which the subtraction strategy was used in each session (the difference between the highest and lowest numerosities on which each student used the strategy). The mean range of numerosities increased from 10 in Practice Session 1 (89-99 blocks) to 12 in Practice Session 2 (87-99 squares) to 16 in Practice Sessions 3 and 4 (83-99 blocks), $F(3,24)=5.49, p=005$, $\eta^{2}=41$. The range of application was significantly greater in Practice Sessions 3 and 4 than in Practice Session 1.

We also examined whether the subtraction strategy came to be used increasingly consistently in the range of numerosities on which it was used at all. To do so, we calculated percentage use of the subtraction strategy on trials with numerosities in between the highest and lowest ones on which the strategy was used in each session. A one-way ANOVA revealed a main effect of session, $F(3,15)=3.79, p<05, \eta^{2}=43$. Consistency of strategy choices in Practice Session 1 was lower than in Practice Sessions 2 and 4 (79\% vs. 98 and $98 \%$, respectively), and consistency in Practice Session $3(93 \%)$ did not differ from that in any of the other sessions.

\section{Solution times}

A 4 (Practice Session: 1, 2, 3, or 4) × 4 (Numerosity: 80-84, 85-89, 90-94, or 95-99) ANOVA on subtraction strategy solution times among children who used the subtraction strategy in all four numerosity ranges in all four practice sessions yielded main effects of session, $F(3,6)=5.86, p<05, \eta^{2}=75$, and numerosity, $F(3,6)=26.25, p<001$, $\eta^{2}=93$. Time needed to execute the subtraction strategy decreased from Practice Session 1 (15.27 s) to Practice Sessions 3 and 4 (10.62 and $10.82 \mathrm{~s}$, respectively). When children used the subtraction strategy, they quantified 95 to 99 blocks (6.68 s) and 90 to 94 blocks $(9.85 \mathrm{~s})$ more rapidly than they did 85 to 89 blocks $(15.78 \mathrm{~s})$ and 80 to 84 blocks $(17.58 \mathrm{~s}$.).

\section{Accuracy}

A parallel Practice Session $\times$ Numerosity ANOVA on the percentage of correct quantifications showed marginally significant effects of numerosity, $F(3,9)=3.51, p=06$, $\eta^{2}=54$, and of the Practice Session $\times$ Numerosity interaction, $F(9,27)=2.18, p=06$, $\eta^{2}=42$. Children generated $41 \%$ correct answers in the range 80 to $84,46 \%$ in the range 85 to $89,68 \%$ in the range 90 to 94 , and $70 \%$ in the range 95 to $99 .{ }^{4}$ In Practice Sessions 1 and 2, percentage correct was greater for grids with 95 to 99 blocks ( 56 and $75 \%$, respectively) and 90 to 94 blocks ( 70 and $75 \%$, respectively) than for grids with 80 to 84 blocks

\footnotetext{
${ }^{4}$ These seemingly low accuracy scores are due to the way in which accuracy was measured in the current study. Only items for which children determined the exact number of colored cells were scored as correct. So, as soon as a response already deviated one block from the correct numerosity, it was scored as incorrect. Taking into account that children's counting skills were still under development and that they were in the midst of exploring the number range up to 100 , it is not surprising that they erred rather frequently when determining numerosities in this range, resulting in a relatively low number of correctly solved trials.
} 
(19 and 31\%, respectively). Percentage correct did not vary with numerosity in Sessions 3 and 4.

\section{Individual paths of development}

The microgenetic design, together with the large number of practice session grids that favored use of the subtraction strategy in the facilitative problems group, allowed indepth analyses of the paths of change followed by individual children in that group. Practice session performance of 14 of the 15 children in this group who ever used the subtraction strategy fell into one of four patterns that differed in the rate and breadth of learning (Fig. 5). Three children (20\% of the group) were rapid and broad learners; they used the subtraction strategy on at least $75 \%$ of trials on all four numerosity ranges (80-84, 85-89, 90-94, and 95-99) in all four practice sessions. This pattern resembles that of adults whose performance was examined in previous studies (e.g., Verschaffel et al., 1998). Five children (33\% of the sample) were broad learners; they did not use the subtraction strategy much on all four numerosity ranges in Practice Session 1 but

a

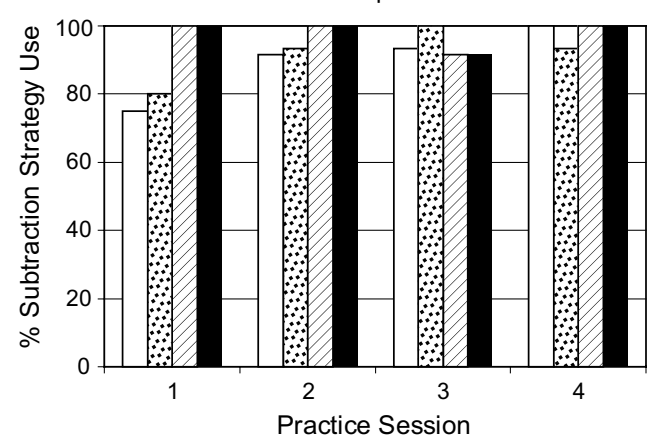

b

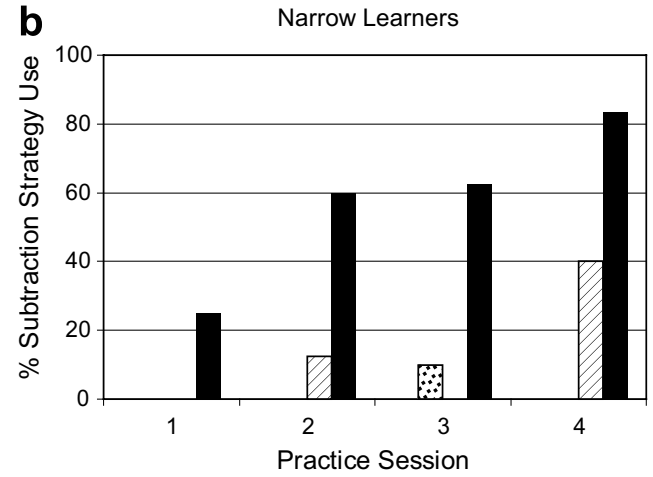

$80-84$

85-89
C

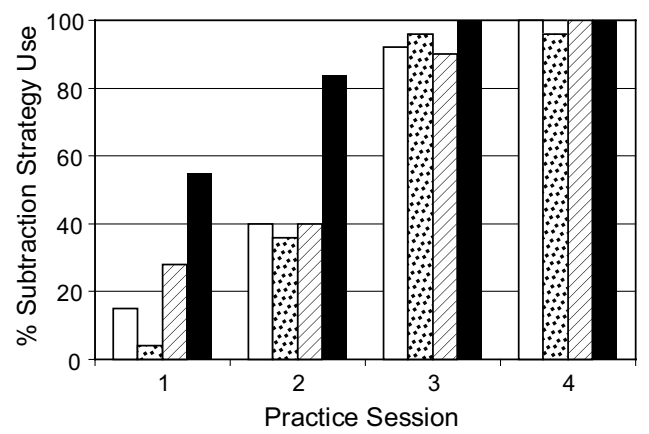

d

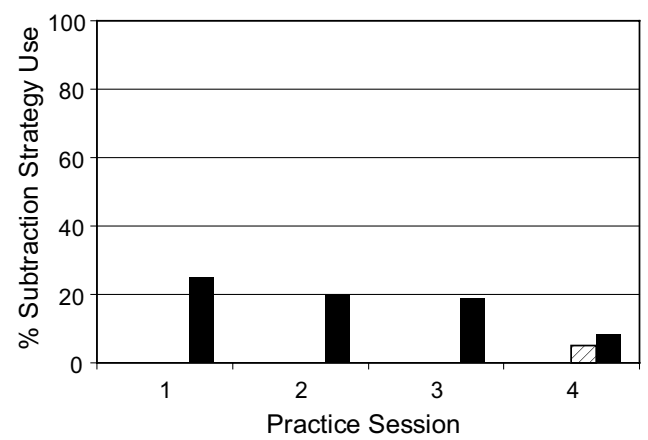

$\square 90-94$

95-99

Fig. 5. Mean paths of development of broad and rapid learners (a), broad learners (b), narrow learners (c), and minimal learners (d). 
did so later. Two children (13\% of the sample) were narrow learners; they used the subtraction strategy on more than $50 \%$ of trials in at least two sessions on the most facilitative problems (those with 95-99 blocks) and at least twice as often across the four sessions in the range 95 to 99 as in any other range. The final four children $(27 \%$ of the sample) were minimal learners; they used the subtraction strategy on fewer than $10 \%$ of trials and never used it on a majority of trials at any numerosity. The one child who did not meet the criteria for any group never used the subtraction strategy until Practice Session 4 but used it quite broadly in that session (on at least $80 \%$ of trials with 85-89, 90-94, or 95-99 blocks).

\section{Discussion}

The current study focused on the discovery and early development of an insightful problem-solving strategy. Combining a microgenetic design with trial-by-trial analyses allowed us to identify the exact point in learning when children first used the subtraction strategy, and this in turn made possible precise analyses of how children systematically extended the new strategy to other problems beyond the initial use. None of the main findings could have emerged from a standard cross-sectional design that averaged data over trials. The findings provided unusually clear information regarding the path, breadth, rate, variability, and sources of change in use of the new problem-solving approach and also hold a number of more general implications for understanding of strategy acquisition.

\section{Path of change}

Perhaps the most striking finding of the study concerned the path of change. Children in both groups began the experiment using the addition strategy; this was not surprising because only children who used this approach consistently in Test Session 1 participated further. More surprising was the next step in the path of change, where $100 \%$ of children in both groups first used the subtraction strategy on an item with 93 to 99 blocks. In the nonfacilitative problems group, items in this range constituted only $7 \%$ of total items, and none in the first 14 trials, so the invariant discovery of the subtraction strategy on problems with very few empty spaces was not attributable to chance.

After the discovery of the subtraction strategy on these nearly complete grids, the path of change involved children progressively extending the strategy to grids with increasing numbers of empty spaces. The extension process was impressively systematic. Children's first use of the subtraction strategy was earlier on grids with 95 to 99 squares than on grids with 90 to 94 squares, earlier on those than on grids with 80 to 89 squares, and earlier on those than on grids with 60 to 79 squares.

The distance that children traveled along this path was quite limited, however, especially given the large advantages that the subtraction strategy conveyed on all of these problems. On problems with 80 to 89 squares, for example, children were more than 1 min faster when they used the subtraction strategy than when they used the addition strategy, and they were considerably more accurate as well. Yet even in Test Session 3, after being presented with 88 problems with 80 to 99 filled squares, children in the 
facilitative problems group still used the subtraction strategy on fewer than half of trials with 80 to 89 blocks.

These data on the path of change on the grid task have implications for understanding strategy choice more generally. Previous studies have identified a strategy's accuracy and speed in the experimental situation as the main influences on how often the strategy is chosen (Geary, 2006; Geary \& Brown, 1991; LeFevre, Smith-Chant, Hiscock, Dale, \& Morris, 2003). Computational models of strategy choice have also emphasized the influence of speed and accuracy in the experimental setting (e.g., Siegler \& Shipley, 1995; Siegler \& Shrager, 1984).

The current findings suggest another factor that needs to be considered to understand many strategy choices, namely, prior experience with the strategies outside the laboratory context. The grid task that was used in the current experiment was in all likelihood unique in the children's experience. However, all of the children would have used addition and counting forward innumerable times to solve problems in other contexts. The children's histories also would have included use of subtraction and counting backward, but these experiences almost certainly would have been far less numerous and probably less successful. Preschoolers count forward before they count backward (Siegler \& Shrager, 1984), first graders receive addition instruction before subtraction instruction, and second graders, such as those in the current experiment, solve a higher percentage of addition problems than inverse subtraction problems (Siegler, 1989). Unfortunately, we have no specific data about children's prior experience in counting forward and backward and in addition and subtraction.

Related findings of experience with strategies in one context influencing strategy choice in other contexts abound in studies of mathematical cognition. To cite three examples, knowledge of addition influences number matching (Bisanz \& LeFevre, 1990), knowledge of addition influences multiplication (Miller \& Paredes, 1990), and knowledge of whole number arithmetic influences decimal fraction arithmetic (Byrnes $\&$ Wasik, 1991). These findings and the current ones suggest that in situations where strategies have extensive histories of use in other situations, models of strategy choice need to include the likely history of the strategies in other contexts as influences on strategy choices in the new context.

Another interesting issue for further research is the effect of children's prior mathematics instruction on their performance on tasks like the one used in the current study. It seems likely that children who have followed a reform-based instructional program that emphasizes the integrated mastery and flexible use of procedural and conceptual knowledge would discover the subtraction strategy earlier and generalize it more extensively than would children who have received purely skills-based instruction focusing on the routine mastery of arithmetic facts and procedures (Hatano, 2003; Verschaffel, Greer, \& De Corte, 2007). The issue seems worthy of empirical test.

\section{Rate of change}

Results from this study underline the value of distinguishing between the "rate of discovery" and the "rate of uptake" of a new strategy. In making this distinction, Siegler (2006) noted that the two rates are only weakly related. Children fairly often discover a strategy after minimal experience but then increase their use of it only slowly. Other times, 
they require extensive experience before they discover a new strategy but then quickly come to use it consistently.

In the current study, the rate of discovery of the subtraction strategy was very fast if viewed in terms of the number of maximally facilitative problems (those with 93-99 blocks). Children in both experimental groups discovered the subtraction strategy after an average of four or five such trials; for children in the facilitative problems group, this occurred within the first eight trials in Practice Session 1.

The rate of uptake of the subtraction strategy was much slower in both groups. Even after encountering many facilitative problems, children used the subtraction strategy on fewer than half of trials in the ranges 60 to 69,70 to 79 , and 80 to 89 in Test Session 3 . The much greater experience with the addition strategy outside of the laboratory, and perhaps greater cognitive resource requirements of subtraction, seems likely to have produced this slow and incomplete uptake of the subtraction strategy.

\section{Breadth of change}

Initially, children applied the subtraction strategy almost exclusively on problems with 90 to 99 filled squares. Even within that range, they used it more often on grids with 95 to 99 filled squares than on those with 90 to 94 filled squares. This narrow breadth of change continued to be present to a large degree throughout the study. Even in Test Session 3, the subtraction strategy was generalized to only approximately $15 \%$ of problems with 60 to 79 filled squares, a range where the strategy still produced considerably faster and somewhat more accurate performance than did the addition strategy.

The situation was quite different with the problems in the transfer session at the end of the study. There, use of the subtraction strategy was surprisingly high, at least when viewed in terms of the percentages of filled and empty spaces. For example, children used the subtraction strategy on $78 \%$ of $5 \times 10$ grids with 45 to 49 blocks and on $40 \%$ of grids with 40 to 44 blocks. These percentages were slightly greater than those in Test Session 3 with the original $10 \times 10$ grids when the same percentages of filled and unfilled spaces were present despite the children never having encountered the $5 \times 10$ grids previously. Substantial transfer of the subtraction strategy was present in both experimental groups, although it was greater in the facilitative problems group.

These findings raise the issue of why the amount of transfer was so high. Obtaining transfer in amounts comparable to initial learning is unusual, especially when the instruction is not designed specifically to promote transfer (Bransford, Brown, \& Cocking, 1999). One possibility is that children based their strategy choices, in whole or in part, on the number of unfilled spaces rather than on the percentage of unfilled spaces. Previous models of strategy choice (Busemeyer \& Myung, 1992; Payne, Bettman, \& Johnson, 1993; Rieskamp \& Otto, 2006; Siegler \& Shipley, 1995) have been based on the assumption that strategy choices are a function of the relative advantages of the available alternatives. On the grid task, the relative advantage of the addition and subtraction strategies is a function of the percentage of unfilled spaces rather than of the number of them. To make this point concretely, in a $3 \times 3$ matrix, children almost surely would count up rather than down even when there were only five unfilled spaces; in a $30 \times 30$ matrix with five unfilled spaces, they almost surely would do the opposite.

However, strategy choices may reflect literal features of problems as well as relative ones. In the current context, children may have been influenced by the absolute number 
of unfilled spaces as well as by the percentage of them. Viewed from this perspective, use of the subtraction strategy on the transfer task was midway between the percentage of use that would have been expected on the basis of the percentage of unfilled spaces and on the basis of the number of unfilled spaces in the $10 \times 10$ grids in Test Session 3. Additional data are needed to test whether literal features of problems as well as relative advantages of strategies on the problems generally influence strategy choices, but it seems likely that they did so in the current context.

\section{Variability of change}

Children varied substantially in their rate and breadth of change, as was evident in the individual difference categories. The rapid and broad learners discovered the subtraction strategy in the first session, used it consistently from the beginning in the original context of 93 to 99 filled squares, and almost immediately used it consistently on problems with 80 to 99 filled squares. The broad learners eventually used the strategy on the same range of problems but extended it at a slower rate. The narrow learners limited use of the subtraction strategy to the most favorable problems - those with few unfilled squares - and they required several sessions before they used it consistently on them. Finally, the minimal learners did not use the subtraction strategy on even half of items on any type of problem; what little use they showed was limited to the most favorable problems in the final session.

In addition to illustrating the variation in individual children's rate and breadth of change, these data indicate the senses in which children's paths of change did and did not vary. All of the children started by using the addition strategy and then began to use the subtraction strategy on problems where it had particularly large advantages. However, for some children this was the end of the path, whereas others extended it further. Given more practice problems, it seems likely that other children also would have followed this path and extended the subtraction strategy to a greater range of problems.

\section{Source of change}

Presentation of a high number and percentage of facilitative problems was hypothesized to increase use of the subtraction strategy in the practice sessions, the test sessions, and the transfer session. Consistent with this hypothesis, in the practice sessions children in the facilitative problems group discovered the subtraction strategy after fewer items and used it more often thereafter. Also as expected, children in the facilitative problems group used the subtraction strategy on more of the transfer problems.

On the other hand, contrary to expectation, the rate of discovery of the subtraction strategy was not affected by the percentage of highly facilitative problems; it was purely a function of the number of such problems. Large advantages in speed and accuracy were insufficient for problems to trigger discovery of the subtraction strategy; only problems where the advantages were extremely large elicited discoveries. Also contrary to expectation, presenting a high percentage of facilitative problems in the practice sessions did not lead to more frequent use of the subtraction strategy in the test sessions or to faster or more accurate performance in them.

A critical issue concerns the unequal distribution of facilitative problems for Trials 1 to 10 and Trials 11 to 20 of the test sessions. Trials 1 to 10 contained only one facilitative 
problem (81), whereas Trials 11 to 20 contained three facilitative problems $(86,95$, and 97). Exposing children to few facilitative problems (and thus to many problems that facilitated the addition strategy) on Trials 1 to 10 in Test Session 1 may have induced a mental set favoring use of the addition strategy that carried over to Trials 11 to 20. In contrast, an alternative design that exposed children to many facilitative problems on Trials 1 to 10 in Test Session 1 might induce a mental set favoring use of the subtraction strategy. Future studies focusing on the effect of prior items on future strategy choices are needed to test this hypothesis.

Having discussed the current findings in terms of the five dimensions of change, we want to make a final remark concerning the children who did not show any evidence of using the subtraction strategy throughout the whole experiment. Although we regarded these children as children who did not yet possess the subtraction strategy, we cannot exclude the possibility that they already had this particular strategy in their repertoire but were unable to apply it in the number range up to 100. This number range factor might also explain why children in the current study did not generalize the subtraction strategy more extensively on the items from the practice and test sessions that were presented in a $10 \times 10$ grid but were quite able to transfer it toward the items from the transfer test being presented in a $5 \times 10$ grid. This alternative explanation could be tested by adapting a paradigm similar to that used in Dowker's (2003) study on computational estimation, where for different number ranges she compared children's competence in computational estimation with their precise computational skills. Analogously, one could first test children's familiarity with the number domain by testing whether they could count up and down fluently in each of the respective decades between 1 and 100, with special attention being paid to their mastery of decade changes. Next, one could divide these children into different groups according to their performance in each decade. For instance, a child in Group 3 might be able to count up and down fluently up to the range 20 to 30 but not up to the range 30 to 40, whereas a child in Group 6 might be able to count up and down fluently up to the range 50 to 60 but not up to the range 60 to 70 . Then for each group one could present a number of highly facilitative items in a grid that corresponds with the respective counting level (e.g., a child in Group 3 could be presented with 27 green blocks in a $5 \times 6$ grid). According to this alternative account, one could expect that a child who belongs to Group 3 will use the subtraction strategy on an item consisting of only two empty squares in a $5 \times 6$ grid but perhaps not on an item with the same number of empty squares in a $10 \times 10$ grid.

To conclude, the current findings suggest that discovery and generalization of the subtraction strategy were influenced by the prior history of use of the addition/counting up and the subtraction/counting down strategies, not just by the relative speeds and accuracies that these strategies yielded in the experimental situation. The more extensive, and probably more successful, history of solving problems via adding and counting forward seems to have predisposed the second graders in this study to subtract or count backward only when those approaches offered overwhelming advantages. From that base, children cautiously expanded their use of the subtraction strategy but almost never to the degree that would have yielded optimal performance in the experimental situation. The general lesson is that understanding strategy discovery and choice requires not only consideration of the strategies' relative advantages on the task presented in the laboratory but also consideration of likely prior experience with the strategies outside of the laboratory. 


\section{Appendix A}

Overview of the stimuli presented in the different sessions of the non-facilitative problems

\begin{tabular}{|c|c|c|c|c|c|c|c|c|}
\hline \multirow[b]{2}{*}{ Trial } & \multicolumn{8}{|l|}{ Session } \\
\hline & Test 1 & Practice 1 & Practice 2 & Test 2 & Practice 3 & Practice 4 & Test 3 & Transfer \\
\hline \multicolumn{9}{|c|}{ Nonfacilitative problems group } \\
\hline 1 & 8 & 20 & 32 & 8 & 17 & 29 & 8 & 39 \\
\hline 2 & 22 & 19 & 50 & 22 & 49 & 26 & 22 & 46 \\
\hline 3 & 53 & 25 & 42 & 53 & 26 & 2 & 53 & 41 \\
\hline 4 & 16 & 31 & 39 & 16 & 43 & 48 & 16 & 36 \\
\hline 5 & 65 & 86 & 97 & 65 & 83 & 99 & 65 & 44 \\
\hline 6 & 81 & 13 & 2 & 81 & 34 & 46 & 81 & 49 \\
\hline 7 & 36 & 35 & 14 & 36 & 10 & 28 & 36 & \\
\hline 8 & 32 & 40 & 28 & 32 & 12 & 16 & 32 & \\
\hline 9 & 49 & 15 & 6 & 49 & 46 & 30 & 49 & \\
\hline 10 & 4 & 1 & 29 & 4 & 5 & 19 & 4 & \\
\hline 11 & 77 & 11 & 36 & 77 & 30 & 9 & 77 & \\
\hline 12 & 95 & 27 & 37 & 95 & 38 & 18 & 95 & \\
\hline 13 & 86 & 48 & 7 & 86 & 33 & 20 & 86 & \\
\hline 14 & 97 & 3 & 47 & 97 & 45 & 11 & 97 & \\
\hline 15 & 44 & 93 & 85 & 44 & 96 & 81 & 44 & \\
\hline 16 & 58 & 24 & 16 & 58 & 8 & 17 & 58 & \\
\hline 17 & 13 & 41 & 9 & 13 & 41 & 49 & 13 & \\
\hline 18 & 74 & 18 & 21 & 74 & 6 & 36 & 74 & \\
\hline 19 & 29 & 44 & 23 & 29 & 1 & 40 & 29 & \\
\hline 20 & 70 & 4 & 22 & 70 & 3 & 47 & 70 & \\
\hline \multicolumn{9}{|c|}{ Facilitative problems group } \\
\hline 1 & 8 & 88 & 93 & 8 & 93 & 87 & 8 & 39 \\
\hline 2 & 22 & 80 & 95 & 22 & 94 & 94 & 22 & 46 \\
\hline 3 & 53 & 93 & 96 & 53 & 91 & 84 & 53 & 41 \\
\hline 4 & 16 & 87 & 86 & 16 & 85 & 83 & 16 & 36 \\
\hline 5 & 65 & 4 & 23 & 65 & 28 & 26 & 65 & 44 \\
\hline 6 & 81 & 94 & 99 & 81 & 83 & 82 & 81 & 49 \\
\hline 7 & 36 & 96 & 92 & 36 & 82 & 97 & 36 & \\
\hline 8 & 32 & 97 & 82 & 32 & 87 & 93 & 32 & \\
\hline 9 & 49 & 89 & 97 & 49 & 80 & 80 & 49 & \\
\hline 10 & 4 & 90 & 84 & 4 & 81 & 85 & 4 & \\
\hline 11 & 77 & 92 & 85 & 77 & 98 & 81 & 77 & \\
\hline 12 & 93 & 85 & 89 & 93 & 99 & 89 & 93 & \\
\hline 13 & 86 & 86 & 81 & 86 & 95 & 86 & 86 & \\
\hline 14 & 95 & 95 & 91 & 95 & 97 & 92 & 95 & \\
\hline 15 & 44 & 43 & 41 & 44 & 9 & 36 & 44 & \\
\hline 16 & 58 & 82 & 94 & 58 & 86 & 96 & 58 & \\
\hline 17 & 13 & 84 & 87 & 13 & 84 & 90 & 13 & \\
\hline
\end{tabular}


Appendix A (continued)

\begin{tabular}{|c|c|c|c|c|c|c|c|c|}
\hline \multirow[b]{2}{*}{ Trial } & \multicolumn{8}{|c|}{ Session } \\
\hline & Test 1 & Practice 1 & Practice 2 & Test 2 & Practice 3 & Practice 4 & Test 3 & Transfer \\
\hline 18 & 74 & 81 & 83 & 74 & 88 & 88 & 74 & \\
\hline 19 & 29 & 91 & 88 & 29 & 90 & 95 & 29 & \\
\hline 20 & 70 & 99 & 98 & 70 & 89 & 91 & 70 & \\
\hline
\end{tabular}

\section{References}

Adolph, K. E. (1997). Learning in the development of infant locomotion. Monographs of the Society for Research in Child Development, 62 (3, Serial No. 251).

Adolph, K.E., Robinson, S.R., Young, J.W., \& Gill-Alvarez, F. (2007). Is there evidence for developmental stages? Unpublished manuscript.

Amsterlaw, J., \& Wellman, H. M. (2006). Theories of mind in transition: A microgenetic study of the development of false belief understanding. Journal of Cognition and Development, 7, 139-172.

Bisanz, J., \& LeFevre, J. (1990). Strategic and nonstrategic processing in the development of mathematical cognition. In D. F. Bjorklund (Ed.), Children's strategies; Contemporary views of cognitive development (pp. 213-244). Hillsdale, NJ: Lawrence Erlbaum.

Bransford, J. B., Brown, A. L., \& Cocking, R. R. (Eds.). (1999). How people learn: Brain, mind, experience, and school. Washington, DC: National Academy Press.

Busemeyer, J. R., \& Myung, I. J. (1992). An adaptive approach to human decision making: Learning theory, decision theory, and human performance. Journal of Experimental Psychology: General, 121, 177-194.

Byrnes, J. P., \& Wasik, B. A. (1991). Role of conceptual knowledge in mathematical procedural learning. Developmental Psychology, 27, 777-786.

Chen, Z., \& Siegler, R. S. (2000). Across the great divide: Bridging the gap between understanding of toddlers' and older children's thinking. Monographs of the Society for Research in Child Development, 66 (2, Serial No. 261).

Coyle, T. R., \& Bjorklund, D. F. (1997). Age differences in, and consequences of, multiple- and variable-strategy use on a multiple sort-recall task. Developmental Psychology, 33, 372-380.

Demetriou, A., \& Raftopoulos, A. (2004). The what, how, and why of developmental change: The emergence of a new paradigm. In A. Demetriou \& A. Raftopoulos (Eds.), Cognitive developmental change: Theories models and measurement (pp. 1-20). Cambridge, UK: Cambridge University Press.

Dowker, A. (2003). Young children's estimates for addition: The zone of partial knowledge and understanding. In A. J. Baroody \& A. Dowker (Eds.), The development of arithmetic concepts and skills (pp. 243-266). Mahwah, NJ: Lawrence Erlbaum.

Dudal, P. (2001). Leerlingvolgsysteem, Wiskunde, Basisboek 2. Leuven, Belgium: Garant.

Geary, D. C. (2006). Development of mathematical understanding. In Handbook of child psychology (6th ed. In D. Kuhn \& R. S. Siegler (Eds.). Cognition, perception, and language (vol. 2, pp. 777-810). Hoboken, NJ: John Wiley.

Geary, D. C., \& Brown, S. C. (1991). Cognitive addition: Strategy choice and speed-of-processing differences in gifted, normal, and mathematically disabled children. Developmental Psychology, 27, 398-406.

Hatano, G. (2003). Foreword. In A. J. Baroody \& A. Dowker (Eds.), The development of arithmetic concepts and skills (pp. xi-xiii). Mahwah, NJ: Lawrence Erlbaum.

Karmiloff-Smith, A. (1992). Beyond modularity: A developmental perspective on cognitive science. Cambridge, MA: MIT Press.

Kuhn, D. (1995). Microgenetic study of change: What has it told us? Psychological Science, 6, 133-139.

Kuhn, D., Garcia-Milla, M., Zohar, A., \& Andersen, C. (1995). Strategies of knowledge acquisition. Monographs of the Society for Research in Child Development, 60 (4, Serial No. 245).

LeFevre, J. A., Smith-Chant, B. L., Hiscock, K., Dale, K. E., \& Morris, J. (2003). Young adults' strategic choices in simple arithmetic: Implications for the development of mathematical representations. In A. J. Baroody \& A. Dowker (Eds.), The development of arithmetic concepts and skills (pp. 203-228). Mahwah, NJ: Lawrence Erlbaum. 
Lemaire, P., \& Siegler, R. S. (1995). Four aspects of strategic change: Contributions to children's learning of multiplication. Journal of Experimental Psychology: General, 124, 83-97.

Luwel, K., Lemaire, P., \& Verschaffel, L. (2005). Children's strategies in numerosity judgment. Cognitive Development, 20, 448-471.

Luwel, K., Verschaffel, L., Onghena, P., \& De Corte, E. (2000). Children's strategies for numerosity judgment in square grids of different sizes. Psychologica Belgica, 40, 183-209.

Luwel, K., Verschaffel, L., Onghena, P., \& De Corte, E. (2001). Strategic aspects of children's numerosity judgment. European Journal of Psychology of Education, 16, 233-255.

Miller, K. E., \& Paredes, D. R. (1990). Starting to add worse: Effects of learning to multiply on children's addition. Cognition, 37, 213-242.

Miller, P. H., \& Aloise-Young, P. A. (1995). Preschoolers' strategic behavior and performance on a samedifferent task. Journal of Experimental Child Psychology, 60, 284-303.

Miller, P. H., \& Coyle, T. R. (1999). Developmental change: Lessons from microgenesis. In E. K. Scholnick, K. Nelson, S. A. Gelman, \& P. H. Miller (Eds.), Conceptual development: Piaget's legacy (pp. 209-239). Mahwah, NJ: Lawrence Erlbaum.

Payne, J. W., Bettman, J. R., \& Johnson, E. J. (1993). The adaptive decision maker. New York: Cambridge University Press.

Rieskamp, J., \& Otto, P. E. (2006). SSL A theory of how people learn to select strategies. Journal of Experimental Psychology: General, 135, 207-236.

Schauble, L. (1996). The development of scientific reasoning in knowledge-rich contexts. Developmental Psychology, 32, 102-119.

Schlagmüller, M., \& Schneider, W. (2002). The development of organizational strategies in children: Evidence from a microgenetic longitudinal study. Journal of Experimental Child Psychology, 81, 298-319.

Siegler, R. S. (1989). Hazards of mental chronometry: An example from children's subtraction. Journal of Educational Psychology, 81, 497-506.

Siegler, R. S. (2006). Microgenetic analyses of learning. In Handbook of child psychology (6th ed.. In D. Kuhn \& R. S. Siegler (Eds.). Cognition, perception, and language (vol. 2, pp. 464-510). Hoboken, NJ: John Wiley.

Siegler, R. S., \& Araya, R. (2005). A computational model of conscious and unconscious strategy discovery. In R. V. Kail (Ed.). Advances in child development and behavior (vol. 33, pp. 1-42). London: Elsevier Academic Press.

Siegler, R. S., \& Jenkins, E. A. (1989). How children discover new strategies. Hillsdale, NJ: Lawrence Erlbaum.

Siegler, R. S., \& Shipley, C. (1995). Variation, selection, and cognitive change. In T. Simon \& G. Halford (Eds.), Developing cognitive competence: New approaches to process modeling (pp. 31-76). Hillsdale, NJ: Lawrence Erlbaum.

Siegler, R. S., \& Shrager, J. (1984). Strategy choices in addition and subtraction: How do children know what to do? In C. Sophian (Ed.), The origins of cognitive skills (pp. 229-293). Hillsdale, NJ: Lawrence Erlbaum.

Siegler, R. S., \& Stern, E. (1998). Conscious and unconscious strategy discoveries: A microgenetic analysis. Journal of Experimental Psychology: General, 127, 377-397.

Verschaffel, L., De Corte, E., Lamote, C., \& Dherdt, N. (1998). The acquisition and use of an adaptive strategy for estimating numerosity. European Journal of Psychology of Education, 13, 347-370.

Verschaffel, L., Greer, B., \& De Corte, E. (2007). Whole number concepts and operations. In F. K. Lester (Ed.), Second handbook of research on mathematics teaching and learning (pp. 557-628). Greenwich, CT: Information Age. 